\title{
Systematic diagonal and vertical errors in antisaccades and memory-guided saccades
}

\author{
Mathias Abegg \\ University of Bern \\ Hyung Lee \\ Keimyung University \\ Jason J S Barton \\ University of British Columbia
}

\begin{abstract}
Studies of memory-guided saccades in monkeys show an upward bias, while studies of antisaccades in humans show a diagonal effect, a deviation of endpoints toward the $45^{\circ}$ diagonal. To determine if these two different spatial biases are specific to different types of saccades, we studied prosaccades, antisaccades and memory-guided saccades in humans. The diagonal effect occurred not with prosaccades but with antisaccades and memory-guided saccades with long intervals, consistent with hypotheses that it originates in computations of goal location under conditions of uncertainty. There was a small upward bias for memory-guided saccades but not prosaccades or antisaccades. Thus this bias is not a general effect of target uncertainty but a property specific to memory-guided saccades.
\end{abstract}

Keywords: Saccade, bias, accuracy

Experimental saccadic paradigms have established a number of contexts that manipulate the relationship between the stimulus and the saccade, in order to vary the involvement of additional factors such as working memory, inhibition, and volitional control. Thus while prosaccades simply require the subject to look at a stimulus that suddenly appears, an antisaccade requires them to make the saccade towards a mirror location in the opposite direction (Hallett, 1978; Hallett \& Adams, 1980; Munoz \& Everling, 2004). While prosaccades require subjects to look at the stimulus as soon as it appears, memory-guided saccades require them to wait for a variable period after the stimulus disappears, until a go signal releases them from fixation (Funahashi, Bruce, \& Goldman-Rakic, 1990).

All types of saccades are associated with a degree of error in their spatial accuracy. These can be divided into variable and systematic errors (White, Sparks, \& Stanford, 1994). Variable error is a random inconsistency in the saccadic endpoint from trial to trial, and likely reflects factors such as uncertainty in the sensory signals indicating goal location (Van Beers, 2007) and signal/noise ratio. Variable error differs among the different types of saccades, being least for prosaccades and greater for antisaccades (Hallett \& Adams, 1980; Krappmann, 1998) and memory-guided saccades (Abel \& Douglas, 2007).

Systematic error is a bias in endpoint that is apparent as a deviation between the goal and the mean saccadic endpoint over many trials (Gnadt, Bracewell, \& Andersen, 1991; White, et al., 1994). A systematic error indicates a more pervasive problem in the estimate of the coordinates of the goal of the saccade. That is, while a hypothetical saccadic performance characterized by low systematic error but high variable error indicates an accurate mean estimate of the goal that is corrupted in individual trials by noise and uncertainty, a performance characterized by high systematic error but low variable error indicates an underlying biasing error in calculating the goal, despite a high-fidelity signal and low uncertainty. Whether systematic and variable errors in the saccadic system arise from the same or different processes is unclear. For memory-guided saccades, one study found similar temporal profiles in the accumulation of variable and systematic errors (Gnadt, et al., 1991), while another reported a dissociation between these two temporal profiles (White, et al., 1994). 
Systematic errors are well known in the saccadic system, particularly for amplitude. Large prosaccades are hypometric, undershooting their goal by about $10 \%$ (Becker, 1989), and memory-guided saccades and antisaccades are more hypometric than prosaccades (Hallett \& Adams, 1980; Krappmann, 1998; Lueck, Tanyeri, Crawford, Henderson, \& Kennard, 1990; Tatler \& Hutton, 2007). Less studied are systematic errors in saccadic direction. Two main directional biases have been described. First, there is an upward bias for memoryguided saccades, in that the endpoints of these eye movements tend to land higher than their intended target (Gnadt, et al., 1991; Stanford \& Sparks, 1994; White, et al., 1994). This has been documented mainly in welltrained research monkeys: the one study that examined this in a few human subjects produced mixed results (Gnadt, et al., 1991). Second, we recently reported a 'diagonal effect' for antisaccades in naïve human subjects (Koehn, Roy, \& Barton, 2008). In this effect, antisaccades directed to locations within the quadrants - rather than the more typically used locations on the horizontal or vertical meridians - show a deviation of their endpoints towards the $45^{\circ}$ diagonal meridians in each quadrant. This diagonal effect was not observed with prosaccades, but has also been reported with manual reaching movements (Gourtzelidis, Smyrnis, Evdokimidis, \& Balogh, 2001; Mantas, Evdokimidis, \& Smyrnis, 2008; Smyrnis, Gourtzelidis, \& Evdokimidis, 2000; Theleritis, Smyrnis, Mantas, \& Evdokimidis, 2004) and in other experiments requiring subjects to make perceptual estimates of the position of stimuli at oblique locations in their visual field (Huttenlocher, Hedges, \& Duncan, 1991; Mantas, et al., 2008; Smyrnis, Mantas, \& Evdokimidis, 2007).

It is not clear why these different studies report two distinctly different systematic biases, an upward bias in monkey memory-guided saccades and a diagonal effect in human antisaccades. It may be that this reflects a species difference, since the upward bias has been reported mainly in monkey experiments, while the diagonal effect was found in a human study. It may be an effect of training, since monkeys tend to be overtrained on saccade tasks, while our human study used mainly naïve subjects. Last, it could reflect differences between the type of programming performed for memory-guided saccades and that for antisaccades. However, the appearance of a discrepancy may simply reflect the fact that no one study has examined for both types of systematic errors, in both antisaccades and memory-guided saccades. To clarify this issue, we performed experiments in humans contrasting prosaccades, antisaccades and memory-guided saccades, using identical stimuli and goal locations, from which we calculated both vertical biases and diagonal effects.

\section{Methods}

\section{Subjects:}

Two experiments were conducted. In the first experiment, there were 10 healthy participants (4 female, age range 26 to 44 years). The data from one subject was excluded due to excessive errors on the antisaccade task. In the second experiment there were 9 healthy participants ( 6 female, age range 22 to 66 years), one of whom had also participated in the first experiment. All subjects tested had normal or corrected-to-normal vision. The protocol was approved by the institutional review boards of Vancouver Hospital and the University of British Columbia, and all subjects gave informed consent in accordance with the declaration of Helsinki.

\section{Apparatus and procedure:}

Subjects were seated in standard dim illumination 57 cm away from a CRT screen, which was covered by a black cardboard mask with a central round window of 30 $\circ$. Head position was maintained by chin and forehead rest. The Eyelink 1000 system (SR Research Ltd., Mississauga, Canada) was used to record movements of the left eye. Stimuli and trials were created using SR Research Experiment Builder 1.1.2, and presented on a NEC Multisync FE 2111SB monitor at $85 \mathrm{~Hz}$. Calibration was performed on a nine point grid at the beginning of the experiment, and considered acceptable if each of the nine points showed an error of less than $1^{\circ}$ of visual angle upon validation. The validity of calibration was tested again before each experimental block and the calibration procedure repeated if a drift of more than $1^{\circ}$ had occurred.

In Experiment 1, there were three experimental blocks, for prosaccades, antisaccades and memory-guided saccades separately. In the prosaccade block, subjects 
were instructed to look at the stimulus as rapidly and accurately as possible. In the antisaccade block, they were to look at a location of the same eccentricity but in the opposite direction (i.e. differing in direction from that of the stimulus by $180^{\circ}$ ). In the memory guided saccade block, subjects were to do a prosaccade to a remembered stimulus location. Each trial began with a fixation display, which showed a white fixation cross at the center of a black screen. After $750 \mathrm{~ms}$, the cross disappeared and the stimulus appeared, a disk with a diameter of $1^{\circ}$. In the prosaccade and the antisaccade trials, the white stimulus remained on the screen for $850 \mathrm{~ms}$ further after saccade onset. The stimulus was then replaced by the fixation display, and the next trial began. For the memory guided saccades, grey rather white stimuli were used. They were presented during $300 \mathrm{~ms}$, followed by a arey blank screen for $300 \mathrm{~ms}$. This was done to avoid afterimages. The blank screen was followed by a fixation light that disappeared after 1.7 seconds; resulting in a 2-second memory period. Subjects were instructed to maintain fixation until the fixation light disappeared.

There were 24 different possible stimulus locations, each at a radial eccentricity of $11.4^{\circ}$ of visual angle. These were regularly spaced, separated from each other by $15^{\circ}$ in terms of direction, with four locations placed on the horizontal or vertical meridians. In all blocks the order of stimulus locations was randomized. Each experimental block consisted of 120 trials ( 5 for each stimulus location), for a total of 360 trials.

In Experiment 2, the apparatus and stimuli were the same as for experiment 1 . There was a single experimental block of memory-guided saccades, but now with a memory period of 6 seconds rather than 2 seconds. The number of trials was the same as in the memory-guided saccade block of experiment 1.

\section{Analysis:}

Data was obtained using SR Research Data Viewer 1.7.5, at a sampling rate of 1000 samples/s. Saccades were detected when eye velocity reached $31 \%$ sec, acceleration exceeded $9100^{\circ} / \mathrm{sec}^{2}$, and position changed by more than $0.15^{\circ}$. The first saccade larger than $1.5^{\circ}$ after stimulus onset was considered the saccadic response. Reaction time was calculated as the time from stimulus onset to saccadic onset (i.e., - saccade of $1.5^{\circ}$ ). Saccades with reaction time less than $80 \mathrm{~ms}$ (considered anticipa- tory eye movements) and more than $800 \mathrm{~ms}$ (considered delayed movements) were excluded from further analysis. If the first eligible saccade in a trial started from a point more than $2^{\circ}$ from screen center the trial was also discarded. Because healthy subjects make a saccade towards the stimulus rather than the goal on about $10 \%$ of antisaccade trials (Everling \& Fischer, 1998; Hutton \& Ettinger, 2006), we excluded trials with large directional errors, namely saccades which an angular error in direction of more than $30^{\circ}$ (Koehn, et al., 2008). Omitted eye movements constituted $3 \pm 1 \%$ (mean \pm s.e.m.) of prosaccade trials, $16 \pm 5 \%$ of antisaccades trials and $10 \pm 3$ $\%$ of memory-guided trials in Experiment 1, and $16 \pm 4$ $\%$ of trials in Experiment 2.

Statistical analysis was done using ANOVA with a general linear model in JMP version 7.0.2 (www.jmp.com). Individual comparisons were examined with the Tukey's honestly significant difference (HSD) test at an $\alpha$-level of 0.05 and quantified with linear contrasts. Mean values from experiment 1 and experiment 2 were compared with the unpaired student's t-test.

\section{Diagonal effect:}

We collapsed the data across both vertical and horizontal hemifields, by inverting the sign of the vertical positional data for saccades to lower hemifield goals, and inverting the sign of the horizontal positional data for saccades to left hemifield goals. Apart from Figure 1, which shows the data for all quadrants, the data in the remaining figures of the results are thus displayed as though all trials were directed into the right upper quadrant. While collapsing the data may obscure any other quadrant- or hemifield-specific biases, these were not the object of our study. Given the relatively few saccades made to any one location, which was necessary to keep the experiment a manageable length while allowing us to compare three saccade types within subjects, the collapsing of data allowed us to increase the number of trials for location of interest to 20 and thereby increase statistical power, in accordance with the methods in our previous study (Koehn, et al., 2008).

For each trial we defined the vectors for the goal and the saccade, where the goal vector was the vector from screen center to the goal location, and the saccade vector was that from screen center to the saccade endpoint. Angular error was calculated as the difference between the 
angles of the goal and saccade vectors. By convention we defined a negative angular error as one where the saccade vector was closer to the horizontal meridian than the goal vector.

We created a trial classification that would allow a specific analysis to reveal a deviation of saccadic endpoints towards the diagonal meridian. First, saccades to goals on the horizontal, vertical or $45^{\circ}$ diagonal meridians were omitted as these are irrelevant for the diagonal effect (Koehn, et al., 2008). (Note that because goals on the horizontal and vertical meridians are equidistant to the two diagonal meridians, one cannot classify a deviation in their saccades as being towards or away from the closest diagonal meridian.) We then classified each of the remaining obliquely directed trials by two aspects of their relation to the diagonal meridian. First is the question of whether they were nearer to the vertical meridian or nearer to the horizontal meridian. For trials in the right upper quadrant, those nearer to the vertical meridian would be 'above' the diagonal, while those nearer to the horizontal meridian would be 'below' the diagonal. If a diagonal effect exists, we should find that the 'above diagonal' trials have a negative angular error, while the 'below diagonal' trials have a positive angular error. This above and below diagonal categorization was used for all our data, which were all reflected into the right upper quadrant for classification for statistical analysis, under the rubric of 'diagonal relation'. Hence trials with goal directions of $60^{\circ}$ or $75^{\circ}$ were 'above diagonal' trials, while trials with goal directions of $15^{\circ}$ or $30^{\circ}$ were 'below diagonal' trials. The second category was 'diagonal proximity' which simply reflected whether the goal location was nearer to the diagonal meridian or to one of the cardinal meridians. Hence we classified trials as 'near to diagonal' $\left(30^{\circ}\right.$ or $\left.60^{\circ}\right)$ or 'far from diagonal' $\left(15^{\circ}\right.$ or $\left.75^{\circ}\right)$.

In experiment 1 we analyzed the data for directional angular error using a general linear model ANOVA with main factors of saccade type (prosaccade, antisaccade, and memory-guided saccade), 'diagonal relation' (above vs. below diagonal), and 'diagonal proximity' (near vs far from diagonal), with subject as a random effect. A diagonal effect in this analysis would be revealed as a significant main effect of or interaction involving diagonal relation. In experiment 2 the general linear model included factors of diagonal relation and diagonal proximity.

\section{Upward bias.}

To determine if any vertical bias existed, we calculated the vertical hypometric error of each saccade. For this we subtracted the absolute vertical position of each saccadic endpoint from the absolute vertical position of each goal. The resulting sign convention was such that a positive value indicated that the vertical component of the saccadic vector was smaller than that for the goal vector (a hypometric error), regardless of the vertical hemifield involved. If an upward bias in saccade endposition exists, this should be reflected in two findings. First, for goals in the upper hemifield, there should be very minimal vertical hypometric error, or possible a negative one, if upward bias was great enough to cauase upward saccades to be hypermetric. Second, for the equivalent mirrorsymmetric locations in the lower hemifield, there should be a larger vertical hypometric error, as an upward bias would compound hypometria to downward locations. Therefore in experiment 1 we subjected vertical hypometric error to a general linear model ANOVA, with main factors of saccade type (prosaccade, antisaccade, memory-guided saccade), and hemifield (upper, lower), with subjects as a random effect. If an upward bias exists, we should find a main effect or interaction involving hemifield, due to smaller hypometric (or even hypermetric) errors for saccades directed into the upper hemifield, compared to larger hypometric errors for saccades into the lower hemifield. For experiment 2, we subjected vertical hypometric error to a general linear model ANOVA with the main factor of hemifield, and subjects as a random effect.

\section{Results}

\section{Diagonal effect:}

Analysis of the angular error in experiment 1 showed a main effect for diagonal relation $(F(1,1940)=18.93$, $\mathrm{p}<0.001)$. The angular error differed for saccades directed to goals 'above' the diagonal meridian compared to saccades directed to goals 'below' the diagonal meridian. There was no main effect of either saccade type $(\mathrm{F}(2,1941)=2.12, \mathrm{p}=0.1196)$ or diagonal proximity $(\mathrm{F}(1,1940)=0.83, \mathrm{p}=0.3621)$. Of the interactions only that between saccade type and diagonal relation was sig- 
nificant $(\mathrm{F}(2,1940)=14.1965, \mathrm{p}<0.0001)$. Tukey's HSD test showed a significant difference between locations 'above' versus 'below' the diagonal for antisaccades $(\mathrm{F}(1,1940)=44, \mathrm{p}<0.0001)$ : antisaccades to goals 'above' the diagonal had a negative angular error $\left(-4.13 \pm 2.14^{\circ}\right.$; mean \pm standard error of the mean) and antisaccades to goals 'below' the diagonal had positive angular error (1.82 $\pm 1.7^{\circ}$ ), indicating that both were biased toward the diagonal meridian (Figure 2). Neither prosaccades $(\mathrm{F}(1,1940)=0.1150, \mathrm{p}=0.73)$ nor memory-guided saccades $(\mathrm{F}(1,1940)=1.04, \mathrm{p}=0.307)$ showed a significant diagonal effect. The angular error in prosaccades was 0.1 $\pm 0.3^{\circ}$ for goals 'above', and $0.35 \pm 0.6^{\circ}$ for goals 'below' the diagonal meridian. For memory guided saccades, the mean angular error was $1.86 \pm 1.0^{\circ}$ for goals 'above', and $0.85 \pm 0.7^{\circ}$ for goals 'below' the diagonal meridan.

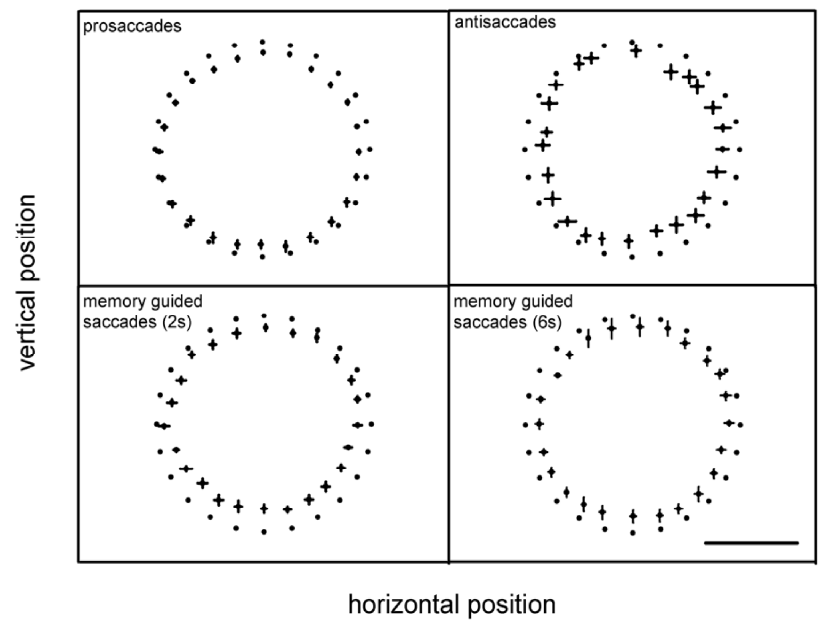

Figure 1. Cartesian plot of mean saccadic endpoints. Saccadic endpoints have error bars that indicate standard error of the mean in the vertical and horizontal directions, and goal locations are shown as black dots without error bars. Endpoints deviate towards the $45^{\circ}$ diagonal for antisaccades, and to a lesser extent for memory-guided saccades with a 6-second memory interval. Prosaccades and antisaccades show a slight downward shift of vertical position whereas memory-guided saccades show an upward bias. Scale bar corresponds to $20^{\circ}$ of visual angle.

To further explore the origins of the interaction between saccade type and diagonal relation, we performed separate ANOVAs for the different saccade types. For antisaccades this confirmed a main effect of diagonal relation on angular error $(\mathrm{F}(1,626)=13.27, \mathrm{p}=0.0003)$. Neither memory-guided saccades nor prosaccades showed a main effect of diagonal relation. Also, none of these ANOVAs showed a main effect of diagonal proximity or an interaction between diagonal relation and diagonal proximity.

Because prior studies have shown that the diagonal effect for memory-guided reaching increases as the interval between stimulus offset and movement onset widened to 4-6 seconds (Mantas, et al., 2008; Smyrnis, et al., 2000), we performed experiment 2, using a 6-second memory period for memory-guided saccades. There was a significant main effect for diagonal relation $(F(1,724)=23.24, p<0.0001)$, but not for diagonal proximity $(F(1,726)=0.61, p=0.4355)$. The mean angular error of memory-guided saccades was $-2.5 \pm 0.9^{\circ}$ to goals above the diagonal, versus $+1.17 \pm 1.0^{\circ}$ to goals below the diagonal. The interaction showed a trend towards significance $(\mathrm{F}(1,726)=3.76, \mathrm{p}=0.0527)$.

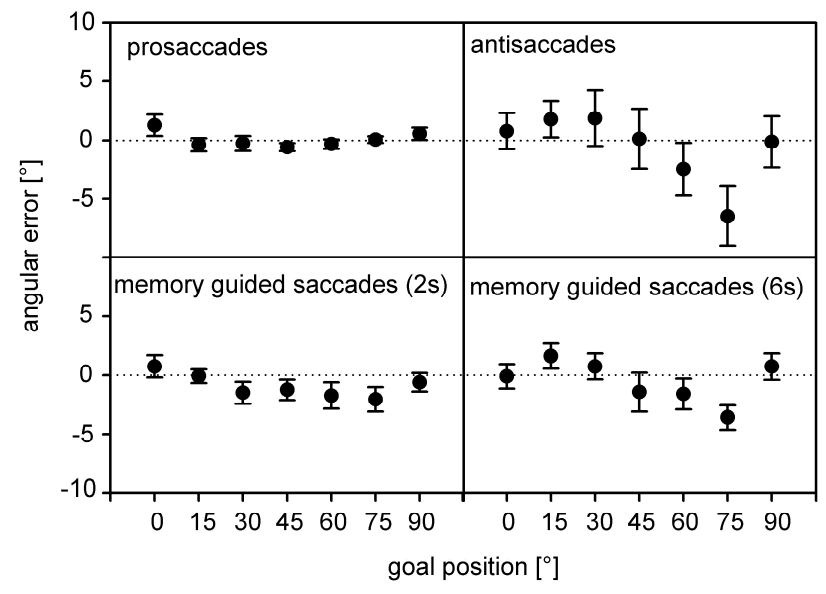

Figure 2. Mean angular error of saccadic endpoints, collapsed into a single quadrant. A positive angular error means the angular direction of the saccade was further away from the horizontal meridian (and closer to the vertical meridian) than the goal vector, whereas a negative angular error means that it was closer to the horizontal meridian. Antisaccades and memory-guided saccades with a 6-second memory interval show more positive angular errors for targets between the horizontal and diagonal meridians $\left(15^{\circ}\right.$ and $\left.30^{\circ}\right)$ and more negative angular errors for saccades between the vertical and diagonal meridians $\left(60^{\circ}\right.$ and $\left.75^{\circ}\right)$, indicating a systematic bias towards the diagonal. Error bars indicate one standard error.

\section{Upward bias:}

For experiment 1 , there was a significant main effect of saccade type on vertical hypometric error $(\mathrm{F}(2,2655)=$ 
32.21, $\mathrm{p}<.0001$ ), and a significant interaction between saccade type and hemifield $(\mathrm{F}(2,2655)=12.65$, $\mathrm{p}<.0001$ ). To explore the origins of this interaction, we performed separate 2-way general linear model analyses on prosaccades, antisaccades and memory-guided saccades, with angular position and hemifield as main factors.

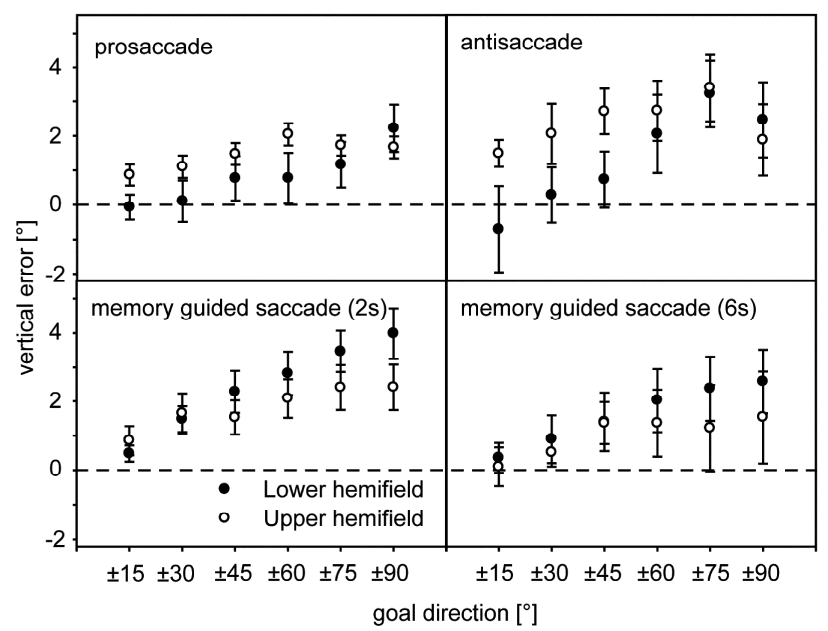

Figure 3. Vertical hypometric error for goals in upper (white discs) versus lower hemifield (black discs). Data is collapsed across the right and left hemifields, but shown for different locations with respect to the horizontal meridian (i.e. $+1-15$ is for goals located $15^{\circ}$ above or below the horizontal meridian). Prosaccades and antisaccades show more vertical hypometric error in the upper than the lower hemifield, indicating a downward bias. Memory-guided saccades show less vertical hypometric error in the upper hemifield, indicating an upward bias. Error bars indicated one standard error.

For memory-guided saccades, there was a main effect of hemifield $(\mathrm{F}(1,971)=13.17, \mathrm{p}=.0003)$, due to saccades being less hypometric in the upper than in the lower hemifield (mean vertical hypometric error in lower hemifield $=1.6 \pm 0.5^{\circ}$, upper hemifield $=1.2 \pm 0.4^{\circ}$ ), consistent with a small upward bias for memory-guided saccades (Figure 3).

For prosaccades, there was a significant main effect for hemifield $(\mathrm{F}(1,1055)=312.08, \mathrm{p}<0.0005)$, due to a slightly less hypometria in the lower hemifield (mean vertical error in lower hemifield $=0.5 \pm 0.2^{\circ}$, upper hemifield $=1.0 \pm 0.3^{\circ}$ ), the reverse of what was found for memory-guided saccades. For antisaccades, there was a trend to significance for hemifield $(\mathrm{F}(1,878)=3.27$, $\mathrm{p}=0.07)$. As for prosaccades, this was due to a bias towards less hypometria in the lower hemifield (mean vertical error in lower hemifield $=0.4 \pm 0.5^{\circ}$, upper hemifield $=0.9 \pm 0.5^{\circ}$ ).

The data for experiment 2 confirm an upward bias for memory-guided saccades, now with a 6-second memory interval. The general linear model shows a significant main effect on vertical hypometric error of hemifield $(\mathrm{F}(1,1012)=16.06, \mathrm{p}<.0001)$ with saccades to the lower hemifield more hypometric than saccades to the upper hemifield (mean vertical error in lower hemifield $=1.0 \pm$ $0.6^{\circ}$, upper hemifield $=0.3 \pm 1.1^{\circ}$ ).

In summary, these results show a small upward bias of $0.4-0.7^{\circ}$ for memory-guided saccades with either $2 \mathrm{~s}$ or 6 s memory intervals, but if anything a small downward bias of $0.5^{\circ}$ for prosaccades and antisaccades.

\section{Discussion}

Our results first confirmed that a significant diagonal effect occurs for antisaccades but not prosaccades. While this was not found with memory-guided saccades with a 2s memory interval, it emerged when the interval was increased to $6 \mathrm{~s}$. Thus the diagonal effect is not specific to antisaccades but can also be found in memory-guided saccades. Second, the analysis of vertical hypometric error showed a small upward bias for memory-guided saccades at both 2-second and 6-second intervals, but not for prosaccades or antisaccades, both of which showed an opposite tendency, towards a downward bias.

\section{Diagonal effect:}

We recently reported that a diagonal effect could be found for antisaccades but not prosaccades, across a variety of experimental paradigms (Koehn, et al., 2008). This diagonal effect is reminiscent of an 'oblique effect' reported for manual reaching movements that require estimation of stimulus position, as when subjects reach in the horizontal plane for stimuli seen in the vertical plane on a visual display, similar to the spatial transforms that occur when we use a computer mouse (Gourtzelidis, et al., 2001; Mantas, et al., 2008; Smyrnis, et al., 2000; Theleritis, et al., 2004). A similar diagonal effect also 
occurs when subjects indicate the position of dots on the circumference of a circle by reproducing the location with a stylus or pen on a different sheet (Huttenlocher, et al., 1991; Mantas, et al., 2008) or using an arrow to indicate the angular direction of the dots (Mantas, et al., 2008; Smyrnis, et al., 2007). These findings and our ocular motor results suggest that the diagonal effect originates in perceptual computations of goal location that are common to a variety of motor responses, particularly when the location of the movement goal has to be estimated rather than directly perceived by the existence of a stimulus located at the movement goal.

Two potential explanations for the diagonal effect have been advanced in the manual reaching and perceptual literature. Common to both is an emphasis on a special status for the cardinal horizontal and vertical axes. The first explanation relates the effect to a general model regarding how uncertain information is handled using categorical classifications and prototypes. It proposes that visual space is classified categorically into quadrants bounded by the horizontal and vertical meridians, in which the central or prototypical direction of each quadrant would be the diagonal axis (Gourtzelidis, et al., 2001; Huttenlocher, et al., 1991). Estimates of the position of obliquely located stimuli, particularly under conditions of uncertainty as in our antisaccade task, would reflect some form of weighted average between the veridical location and the prototype direction. This has some similarity to some explanations of the 'range effect', in which saccadic endpoints tend to deviate towards the mean position of all the locations used in an experimental block (Kapoula, 1985), as the mean position can be considered as a temporary, experiment-specific prototype direction.

The second proposal suggests that the representation of space is non-linear. This explanation proposes that there is an expansion of representations around the cardinal axes, much like the increased emphasis on foveal vision in striate cortex, and a contraction of representations around the diagonal axes (Mantas, et al., 2008). Under conditions of stimulus uncertainty, it has been suggested that this asymmetry may through some unspecified mechanism lead to shifts of localization towards regions of sparser representation. At present, though, we are not aware of any physiological evidence for increased neural representation of space around the cardinal meridians.
Regardless of which explanation is correct, the fact that the diagonal effect may emerge under conditions of positional uncertainty can both explain the lack of such an effect for prosaccades and predict that it would occur for memory-guided saccades too, particularly when the interval between the occurrence of the stimulus and the time of saccade onset increases, as we found. The fact that we find a diagonal effect with memory intervals of 6 seconds but not of 2 seconds may be consistent with a report of a significant effect of the duration of the memory interval in a similar 'oblique effect' for manual reaching movements, using intervals of $0,2,4$ and 6 seconds (Smyrnis, et al., 2000). However, the fact that our two different memory-guided saccade paradigms were performed by different subjects in different sessions limits our ability to confirm an effect of the memory interval.

In the introduction we raised the question as to whether the discrepancies in reports of upward bias in monkey memory-guided saccades and the diagonal effect in human antisaccades reflected a species difference in systematic errors. While we have established that the diagonal effect occurs for both antisaccades and memoryguided saccades in humans, our work obviously cannot establish whether monkeys also show a diagonal effect. Deducing this from the studies that established upward bias in antisaccades is difficult. These used stimuli either on the horizontal, vertical or diagonal meridians (Gnadt, et al., 1991; White, et al., 1994), where a diagonal effect does not exist, or within $15^{\circ}$ of the diagonal meridians (Stanford \& Sparks, 1994; White, et al., 1994), where the diagonal effect is weakest (Koehn, et al., 2008). Furthermore, the large upward bias demonstrated in these studies might have obscured any diagonal effect.

\section{Upward bias:}

On the other hand, our results show that memoryguided saccades are less hypometric when directed towards the upper hemifield instead of the lower hemifield, an effect not seen with prosaccades or antisaccades. These findings are consistent with previous reports of an upward shift of the saccadic endpoint in monkeys doing memory-guided saccades. However, the effect is very small in our human subjects. The difference in vertical error between saccades to stimuli in the upper versus the lower hemifield was only $0.4^{\circ}$ to $0.7^{\circ}$, which indicates an upward bias of $0.2^{\circ}$ to $0.35^{\circ}$. In contrast, well-trained 
monkeys show a substantial upward bias of up to $4^{\circ}$ (Goffart, Quinet, Chavane, \& Masson, 2006; White, et al., 1994), about 10-20 times larger. One possible reason for this difference is related to the observation that in monkeys the upward bias for memory-guided saccades is present in complete darkness but not in bright light (Goffart, et al., 2006). Our experiment was performed in very dim lighting, but visual contours were still slightly discernible: hence having some slight background illumination may have reduced the effect in our subjects. A second possible reason for the difference in magnitude of upward bias is that this represents a species difference, between monkey and human subjects. The only other investigation of vertical bias in human memory-guided saccades studied five subjects on a case-by-case basis: this showed a systematic vertical bias in three of five subjects (Gnadt, et al., 1991). However, in two of these three the bias varied with angular direction of the saccade. In one subject (H52) this appeared to be a general hypermetria, while in another (H53) the bias was downward. Only one subject (H54) showed a consistent upward bias of about 1.3 to $1.8^{\circ}$, also smaller than in their monkeys. The reasons for their between-subject inconsistency are unclear, but may have to do with the use of a very short memory interval of $400 \mathrm{~ms}$, since their own monkey data showed that the upward bias continued to increase as the memory interval widened from $400 \mathrm{~ms}$ to $1000 \mathrm{~ms}$.

A few other studies have also reported on upper and lower hemifield differences in other saccades besides memory-guided saccades. Larger downward than upward antisaccades have been reported in humans and monkeys(Bell, Everling, \& Munoz, 2000; Dafoe, Armstrong, \& Munoz, 2007), as well as slightly less hypometric downward than upward prosaccades in humans (Dafoe, et al., 2007), both of which are similar to what we found, and opposite to the upward bias for memoryguided saccades. Thus, these studies reinforce our conclusion that upward bias is specific for memory-guided saccades.

The origin of upward bias remains speculative. Others have discussed artefactual reasons such as offsets in fixation position or fixation drift (White, et al., 1994). Our practice of excluding saccades with significant deviations of onset position from screen center should eliminate these factors, as did the calibration procedures in the monkey experiments (White, et al., 1994); moreover, a drift should affect all goal angles equally and be found also in the prosaccade and the antisaccade blocks, which was not the case. A neural basis for upward bias in memory-guided saccades was examined in a study that also recorded neural responses in the superior colliculus (Stanford \& Sparks, 1994). This reported that the directional responses of collicular neurons did not show an equivalent shift with the systematic error, suggesting that upward bias originated at a brainstem level distal to the colliculus. If so, one might expect a similar upward bias for responses like antisaccades, since it is unlikely that at the brainstem level the ocular motor mechanisms would differ significantly between antisaccades and memoryguided saccades. Instead, we found a small downward bias for antisaccades, similar to that for prosaccades. This suggests that the upward bias may reflect some key difference in programming ocular motor coordinates that is generated by the dynamics involved when saccadic execution must be delayed, rather than triggered immediately as is the case with prosaccades and antisaccades. Indeed, others have speculated that such directional biases“...may be due to influences from cortical structures related to goals/target selection such as area LIP and the FEF and could consequently represent an adaptation in behavioral strategies" (Bell, et al., 2000). Given the small size of the upward and downward biases we and others (Gnadt, et al., 1991) report in humans, compared to the large biases reported in monkeys (White, et al., 1994), such conjectures await confirmation from data regarding vertical biases in monkey antisaccades and memory-guided saccades.

\section{Acknowledgements}

This work was supported by CIHR grant MOP-81270. MA was supported by "Schweizerische Stiftung für medizinisch-biologische Stipendien". JB was supported by a Senior Scholar Award from the Michael Smith Foundation for Health Research and a Canada Research Chair.

\section{References}

Abel, L. A., \& Douglas, J. (2007). Effects of age on latency and error generation in internally mediated saccades. Neurobiol Aging, 28(4), 627-637. 
Becker, W. (1989). Metrics. In R. H. Wurtz \& M. E. Goldberg (Eds.), The Neurobiology of Saccadic Eye Movements (pp. 13-68). Amsterdam: Amsterdam.

Bell, A. H., Everling, S., \& Munoz, D. P. (2000). Influence of Stimulus Eccentricity and Direction on Characteristics of Pro- and Antisaccades in NonHuman Primates. J Neurophysiol, 84, 2595-2604.

Dafoe, J. M., Armstrong, I. T., \& Munoz, D. P. (2007). The influence of stimulus direction and eccentricity on pro- and anti-saccades in humans. Exp Brain Res, 179(4), 563-570.

Everling, S., \& Fischer, B. (1998). The antisaccade: a review of basic research and clinical studies. Neuropsychologia, 36, 885-899.

Funahashi, S., Bruce, C. J., \& Goldman-Rakic, P. S. (1990). Visuospatial coding in primate prefrontal neurons revealed by oculomotor paradigms. $J$ Neurophysiol, 63(4), 814-831.

Gnadt, J. W., Bracewell, R. M., \& Andersen, R. A. (1991). Sensorimotor transformation during eye movements to remembered visual targets. Vision Res, 31(4), 693-715.

Goffart, L., Quinet, J., Chavane, F., \& Masson, G. S. (2006). Influence of background illumination on fixation and visually guided saccades in the rhesus monkey. Vision Res, 46(1-2), 149-162.

Gourtzelidis, P., Smyrnis, N., Evdokimidis, I., \& Balogh, A. (2001). Systematic errors of planar arm movements provide evidence for space categorization effects and interaction of multiple frames of reference. Exp Brain Res, 139(1), 59-69.

Hallett, P. (1978). Primary and secondary saccades to goals defined by instructions. Vision Res, 18, 1279-1296.

Hallett, P., \& Adams, B. (1980). The predictability of saccadic latency in a novel voluntary oculomotor task. Vision Res, 20, 329-339.

Huttenlocher, J., Hedges, L. V., \& Duncan, S. (1991). Categories and particulars: prototype effects in estimating spatial location. Psychol Rev, 98(3), 352376.

Hutton, S., \& Ettinger, U. (2006). The antisaccade task as a research tool in psychopathology: a critical review. Psychophysiology, 43, 302-313.
Kapoula, Z. (1985). Evidence for a range effect in the saccadic system. Vision Res, 25(8), 1155-1157.

Koehn, J. D., Roy, E., \& Barton, J. J. S. (2008). The 'diagonal effect': a systematic error in oblique antisaccades. . J Neurophysiol, 100, 587-597.

Krappmann, P. (1998). Accuracy of visually and memory-guided antisaccades in man. Vision Res, 38, 2979-2985.

Lueck, C., Tanyeri, S., Crawford, T., Henderson, L., \& Kennard, C. (1990). Antisaccades and remembered saccades in Parkinson's disease. J Neurol Neurosurg Psychiatry, 53, 284-288.

Mantas, A., Evdokimidis, I., \& Smyrnis, N. (2008). Perception action interaction: the oblique effect in the evolving trajectory of arm pointing movements. Exp Brain Res, 184(4), 605-616.

Munoz, D., \& Everling, S. (2004). Look away: the antisaccade task and the voluntary control of eye movement. Nature Reviews Neurosci, 5, 218-228.

Smyrnis, N., Gourtzelidis, P., \& Evdokimidis, I. (2000). A systematic directional error in 2-D arm movements increases with increasing delay between visual target presentation and movement execution. Exp Brain Res, 131(1), 111-120.

Smyrnis, N., Mantas, A., \& Evdokimidis, I. (2007). "Motor oblique effect": perceptual direction discrimination and pointing to memorized visual targets share the same preference for cardinal orientations. J Neurophysiol, 97(2), 1068-1077.

Stanford, T. R., \& Sparks, D. L. (1994). Systematic errors for saccades to remembered targets: evidence for a dissociation between saccade metrics and activity in the superior colliculus. Vision Res, 34(1), 93-106.

Tatler, B. W., \& Hutton, S. B. (2007). Trial by trial effects in the antisaccade task. Exp Brain Res, 179(3), 387-396.

Theleritis, C., Smyrnis, N., Mantas, A., \& Evdokimidis, I. (2004). The effects of increasing memory load on the directional accuracy of pointing movements to remembered targets. Exp Brain Res, 157(4), 518-525.

Van Beers, R. J. (2007). The sources of variability in saccadic eye movements. J Neurosci, 27(33), 87578770 . 
White, J. M., Sparks, D. L., \& Stanford, T. R. (1994).

Saccades to remembered target locations: an analysis of systematic and variable errors. Vision Res, 34(1), 79-92. 